\title{
Some Remarks on Operator- Convex Functions
}

\author{
CHARLES A. McCARTHY*
}

Let $\mathcal{H}$ be a Hilbert space; we denote convergence in the weak and strong operator topologies of $B(\mathcal{F C})$ by $\rightarrow$ and $\rightarrow$. A Borel function $\phi(\lambda)$ defined on the open interval $a<\lambda<b$ is said to be operator-convex on $(a, b)$ if $\phi(A+B / 2) \leqq$ $\frac{1}{2}[\phi(A)+\phi(B)]$ for all self-adjoint $A, B$ with spectra in $(a, b)$. Such $\phi$ have been characterized in a number of ways, but for our purposes the most useful is that of J. Bendat and S. Sherman [1]: $\phi$ is operator-convex on $(a, b)$ if and only if

$$
\phi(\lambda)=r+s \lambda+\int \frac{\lambda^{2} t}{t-\lambda} \mu(d t),
$$

with $\mu$ a finite non-negative measure supported on the complement of $(a, b)$ in $R^{1} \cup\{\infty\}$

We wish to present here two theorems concerning operator-convex functions:

Theorem 1. Let $\phi$ be an operator-convex function on $(a, b)$; let $A_{\alpha}, A$ be self-adjoint with spectra in $(a, b)$. Suppose that $A_{\alpha} \rightarrow A$ and $\phi\left(A_{\alpha}\right) \rightarrow B$. Then $\phi(A) \leqq B$.

Theorem 2. In Theorem 1, suppose further that $\phi(A)=B$. Then either $\phi(\lambda)=$ $r+s \lambda$ or $A_{\alpha}$ in fact converges strongly to $A$.

The considerations involved in these theorems arose in [2] (the fact that $\lambda^{-1}$ is operator-convex on $(0, \infty)$ was used to show that the limit in the weak operator topology of a minimizing sequence of operators is itself a minimizing operator); in some cases, Theorem 2 shows that a weakly convergent minimizing sequence in fact converges strongly. We further hope that these theorems, after being suitably adapted for unbounded operators, may also prove useful in demonstrating some sort of strong convergence as a consequence of weak convergence of their resolvents to the correct limits.

Preliminary to giving the proofs of these theorems, we notice that if $\phi$ is unbounded at $a$ or $b$, then by the Uniform Boundedness Theorem the spectra

* Supported by the National Science Foundation, GP 12631. 
of $A_{\alpha}$ and $A$ must be bounded away from that point; $\phi$ is therefore continuous on the smallest closed interval [ $c, d]$ which contains the spectra of all $A_{\alpha}$ and $A$. The proof of Theorem 1 is immediate: let $C_{\alpha}$ be a convex combination of $\left\{A_{\beta}: \beta \geqq \alpha\right\}-C_{\alpha}=\Sigma_{\beta} c_{\alpha \beta} A_{\beta}$-such that $C_{\alpha} \rightarrow A$. Then $\phi\left(C_{\alpha}\right) \rightarrow \phi(A)$, as may be seen by approximating $\phi$ uniformly by a polynomial on $[c, d]$ and noting that $C_{\alpha}^{k} \longrightarrow C^{k}$ for every $k$. But $\phi\left(C_{\alpha}\right) \leqq \Sigma_{\beta} c_{\alpha \beta} \phi\left(A_{\beta}\right) \rightarrow B$ and hence $\phi(A) \leqq B$.

For the proof of Theorem 2, we may suppose that $r=s=0$ in $\left(^{*}\right)$; we then need only show that if $\mu \neq 0$ then $A_{\alpha}$ converges strongly to $A$.

If $\mu=\mu_{1}+\mu_{2}$ is any decomposition of $\mu$ into the sum of two non-negative measures, set $\phi_{i}(\lambda)=\int \lambda^{2} t(t-\lambda)^{-1} \mu_{i}(d t) . \phi_{1}$ and $\phi_{2}$ are both operator-convex on $(a, b)$. Let $B_{1}$ be any weak cluster point of $\phi_{1}\left(A_{\alpha}\right)$, say $\phi_{1}\left(A_{\beta}\right) \underset{\omega}{\rightarrow} B_{1}$; let $B_{2}$ be any weak cluster point of $\phi_{2}\left(A_{\beta}\right)$, say $\phi_{i}\left(A_{\gamma}\right) \rightarrow B_{i}$. We claim that $\phi_{1}(A)=B_{1}$. If not, we would have (by Theorem 1 ) an element $x$ of $\mathcal{H}$ for which $\left(\phi_{1}(A) x, x\right)<$ $\left(B_{1} x, x\right)$; in any case we have $\left(\phi_{2}(A) x, x\right) \leqq\left(B_{2} x, x\right)$ and thus a contradiction: $(B x, x)=(\phi(A) x, x)=\left(\phi_{1}(A) x, x\right)+\left(\phi_{2}(A) x, x\right)<\left(B_{1} x, x\right)+\left(B_{2} x, x\right)=$ $\lim _{\gamma}\left(\phi\left(A_{\gamma}\right) x, x\right)=(B x, x)$.

Now let $t_{0}$ be some point in the support of $\mu$. If $\mu\left(\left\{t_{0}\right\}\right) \neq 0$, let $\mu_{1}$ above be the restriction of $\mu$ to $\left\{t_{0}\right\}$; we see that for the function $\phi_{0}(\lambda)=\lambda^{2}\left(t_{0}-\lambda\right)^{-1}$ that $\phi_{0}\left(A_{\alpha}\right) \rightarrow \phi_{0}(A)\left[\phi_{0}(\lambda)=\lambda^{2}\right.$ if $\left.t_{0}=\infty\right]$. This is sufficient to show that $A_{\alpha} \rightarrow A$ : define $C_{\alpha}=A_{\alpha}-t_{0}$ or $t_{0}-A_{\alpha}$ whichever be positive (necessarily $t_{0}<c$ or $\left.t_{0}>d\right)$. Then $A_{\alpha} \rightarrow A$ gives $C_{\alpha} \underset{\bullet}{\rightarrow} C$, and $\phi_{0}\left(A_{\alpha}\right) \rightarrow \phi_{0}(A)$ gives $C_{\alpha}^{-1}\left(C_{\alpha} \pm t_{0}\right)^{2} \rightarrow$ $C^{-1}\left(C \pm t_{0}\right)^{2}$ so $C_{\alpha}^{-1} \rightarrow C^{-1}$. Set $D_{\alpha}=C^{-1 / 2} C_{\alpha} C^{-1 / 2}$; the spectra of $D_{\alpha}$ are uniformly bounded away from 0 and $\infty$, and $D_{\alpha} \rightarrow I, D_{\alpha}^{-1} \rightarrow I$. Write the spectral decomposition of $D_{\alpha}$ as $D_{\alpha}=\int \lambda E_{\alpha}(d \lambda)$. From $D_{\alpha}+D_{\alpha}^{-1}-2 I \rightarrow 0$, we have for any $x$ in $\mathfrak{H C}$

$$
\int_{0}^{\infty}\left(\lambda+\lambda^{-1}-2\right)\left(E_{\alpha}(d \lambda) x, x\right) \rightarrow 0
$$

As the integrand and spectral measure are both non-negative for $\lambda>0$, we have also

$$
\int_{0}^{\infty} \lambda\left(\lambda+\lambda^{-1}-2\right)\left(E_{\alpha}(d \lambda) x, x\right) \rightarrow 0 .
$$

Thus $\left\|\left(D_{\alpha}-I\right) x\right\|^{2} \rightarrow 0, D_{\alpha} \rightarrow I, C_{\alpha} \rightarrow C$, and $A_{\alpha} \rightarrow A$. [If $t_{0}=\infty$ then $A_{\alpha}^{2} \rightarrow A^{2}$ gives $\left\|A_{\alpha} x\right\|^{2} \rightarrow\|A \dot{x}\|^{2}$ which with $A_{\alpha} x \rightarrow \dot{A} x$ yields $A_{\alpha} x \rightarrow A x$.]

In the case that $\mu$ is purely continuous, there must be a finite $t_{0}$ in the support of $\mu$ which is neither $a$ nor $b$. Define $\mu_{\mathrm{e}}$ to be the restriction of $\mu$ to the interval $\left(t_{0}-\epsilon, t_{0}+\epsilon\right)$, and $\operatorname{set} \phi_{\epsilon}(\lambda)=\left[\mu\left(t_{0}-\epsilon, t_{0}+\epsilon\right)\right]^{-1} \int \lambda^{2} t(t-\lambda)^{-1} \mu_{\epsilon}(d t)$. We know 
that for each fixed $\epsilon, \phi_{\epsilon}\left(A_{\alpha}\right) \rightarrow \phi_{\epsilon}(A) . \phi_{\epsilon}(\lambda)$ converges uniformly on $[c, d]$ to $\phi_{0}(\lambda)=\lambda^{2} t_{0}\left(t_{0}-\lambda\right)^{-1}$, so $\phi_{0}\left(\ddot{A}_{\alpha}\right) \rightarrow \phi_{0}(A)$, and the proof may be completed as before.

\section{REFERENCES}

[1] J. Bendat \& S. Sherman, Monotone and Convex Operator Functions, Trans. AMS, 79 (1955) 58-71.

[2] C. McCarthy, Optimal Conditioning of Operators on Hilbert Space, Proceedings of the Conference on Functional Analysis and its Applications, Monterey California, 1969.

University of Minnesota

Date communicated: ОстовER 15, 1970 